Mathematical Modelling and Analysis

Volume 21 Number 1, January 2016, 16-34

http://dx.doi.org/10.3846/13926292.2016.1128488

(c) Vilnius Gediminas Technical University, 2016
Publisher: Taylor\&Francis and VGTU

http://www.tandfonline.com/TMMA

ISSN: 1392-6292

eISSN: $1648-3510$

\title{
Application of Collocation BEM for Axisymmetric Transmission Problems in Electro- and Magnetostatics
}

\author{
Olga Lavrova ${ }^{a}$ and Viktor Polevikov ${ }^{b}$ \\ ${ }^{a}$ Faculty of Mechanics and Mathematics, Belarusian State University \\ Independence Ave. 4, 220030 Minsk, Belarus \\ ${ }^{b}$ Faculty of Applied Mathematics and Informatics, Belarusian State University \\ Independence Ave. 4, 220030 Minsk, Belarus \\ E-mail(corresp.): lavrovaolga@mail.ru \\ E-mail: polevikov@bsu.by
}

Received February 26, 2015; revised November 19,2015; published online January 15, 2016

\begin{abstract}
This paper considers the numerical solution of boundary integral equations for an exterior transmission problem in a three-dimensional axisymmetric domain. The resulting potential problem is formulated in a meridian plane as the second kind integral equation for a boundary potential and the first kind integral equation for a boundary flux. The numerical method is an axisymmetric collocation with equal order approximations of the boundary unknowns on a polygonal boundary. The complete elliptic integrals of the kernels are approximated by polynomials. An asymptotic kernels behavior is analyzed for accurate numerical evaluation of integrals. A piecewise-constant midpoint collocation and a piecewise-linear nodal collocation on a circular arc and on its polygonal interpolation are used for test computations on uniform meshes. We analyze empirically the influence of the polygonal boundary interpolation to the accuracy and the convergence of the presented method. We have found that the polygonal boundary interpolation does not change the convergence behavior on the smooth boundary for the piecewise-constant and the piecewise-linear collocation.
\end{abstract}

Keywords: transmission problem, Laplace equation, weakly singular integral equation, boundary element method, axisymmetric collocation, polygonal boundary.

AMS Subject Classification: 35J05; 65N38.

\section{Introduction}

Many problems in electro- and magnetostatics require solution of the Maxwell's equations in a domain, comprised by media of different properties. A linear polarization and a linear magnetization of the media are widely used assumptions in electro- and magnetostatics, respectively. For instance, a linear magnetization of ferrofluids in ferrohydrostatics is a reasonable approximation of a 
nonlinear one in weak magnetic fields or in a narrow range of magnetic field intensities [17]. Field problems for linear materials, formulated in terms of the potential, result in transmission problems for the Laplace equation. In this paper we consider an exterior transmission problem for a bounded nonconducting medium surrounded by another nonconducting medium with a given field at the infinity. This specific formulation is particularly important for problems of control and manipulation of ferrofluid droplets by external magnetic fields, which presents a promising technique for handling samples in biological and chemical systems $[15,18]$. One of the medical application is to target the magnetic drug to the diseased part by means of the external magnetic force.

An application of boundary element methods for three-dimensional transmission problems in electro- and magnetostatic field problems is discussed e.g in $[2,6,14]$. Several boundary integral approaches for the reduced potential are compared with respect to accuracy and computational times in [2]. The authors show, in particularly, that the Steklov-Poincaré operator formulation gives the best approximation. In this paper we consider a direct double layer formulation for the total potential of the magnetostatic field problem with no current density. Test computations, similar to an example on a sphere in [2], are performed to compare the accuracy of the axisymmetric method of the present paper and the three-dimensional methods in [2].

A collocation boundary element method is one of the widely-used numerical methods for solution of boundary integral equations. Beginning in the mid-1970's, collocation solutions of integral equations for axisymmetric problems have been extensively considered in the literature, see e.g. [19, Chapter 6]. However, we did not find in the literature any theoretical result about convergence of collocation methods in the meridian plane. Therefore numerical results in the paper are compared with the existing theory for two-dimensional geometry $[3,7,8,9,16]$.

An axisymmetric collocation boundary element method with the polynomial approximation of the complete elliptic integrals is a well-known technique for solution of the potential problems. An accuracy of this technique, applied to the transmission problem on an approximate polygonal boundary between two media, is in focus of the research. For that, kernels behavior is analyzed and test computations on a sphere are performed. Accurate computations of the boundary unknowns are of high importance for free-surface problems of electroand ferrohydrostatics, where an a-priori unknown moving boundary presents an interface between two media. In this case the boundary element method is applied over the boundary, which itself is a numerical solution of the freesurface subproblem at the every iteration step of the solution process, see e.g. in [11]. The boundary element method, presented in the paper, was extended to a coupling with a finite element method to treat nonlinearities inside of the bounded domain in [11]. The boundary element - finite element method for a magnetostatic subproblem was further coupled with free-surface computations in [11] to find axisymmetric equilibrium shapes of a ferrofluid drop in a free space [12] and in a capillary [13].

An accurate collocation solution of the integral equations on polygonal boundaries in the meridian plane, is in focus of the research. We consider an ex- 
terior transmission problem for the Laplace equation in axisymmetric domains. Two boundary integral equations are formulated in the way, which allows to find the unknown boundary potential and the boundary flux separately in two stages, see Section 2. The second kind integral equation is constructed only for the potential, the first kind integral equation is used to find the boundary flux for the computed boundary potential. The reformulation in the meridian plane leads to weakly singular kernels for both equations, see Section 3. We analyze an asymptotic behavior of the kernel functions for integration points, tending to a source point, to perform an accurate numerical evaluation of integrals. The polygonal boundary allows some simplifications of the kernels, influencing to the behavior near the symmetry axis, see Section 4. Piecewise-constant and piecewise-linear equal order approximations for the unknown functions are applied for test computations, see Section 5. To study an effect of using interpolation to approximate the boundary, we analyze the accuracy and the convergence of the axisymmetric collocation method on a circular arc and on the polygonal boundary interpolation. We compare approximate and exact solutions for the boundary potential and the boundary flux in different norms (Test 1) and compute the accuracy of the boundary field for different values of permeabilities (Test 2).

\section{Exterior transmission problem in 3D}

We consider a magnetostatic problem for a bounded simply connected domain $\Omega$ with a smooth or piecewise-smooth boundary $S$ of a linearly-magnetizable medium, $\boldsymbol{B}_{1}=\mu_{1} \boldsymbol{H}_{1}$ in $\Omega$, surrounded by another medium, $\boldsymbol{B}_{2}=\mu_{2} \boldsymbol{H}_{2}$ in $R^{3} \backslash \bar{\Omega}$. Here $\boldsymbol{B}_{i}$ denotes the magnetic induction, $\boldsymbol{H}_{i}$ - the magnetic field vector, $\mu_{i}$ - the constant permeability. Both media are nonconducting, i.e. $\nabla \times \boldsymbol{H}_{i}=\mathbf{0}$. Far from the domain $\Omega$ we have a fixed region containing a source of a static magnetic field $\boldsymbol{H}_{\text {ext }}$ - such as a current-carrying coil, permanent magnets or other materials. The external field $\boldsymbol{H}_{\text {ext }}$ is defined at the infinity for the model under study, see Figure 1.

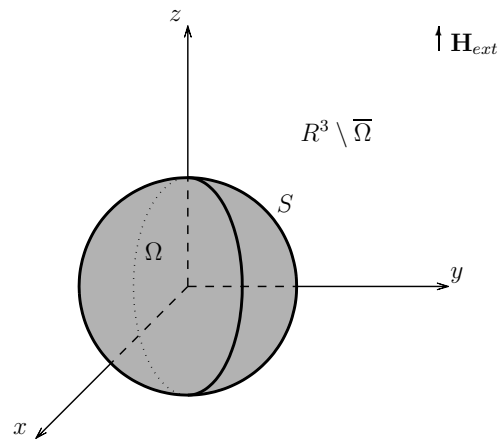

Figure 1. The problem setting.

The mathematical model in terms of the magnetostatic potentials $u_{1}$ and 
$u_{2}$, see [17], consists of the Laplace equations

$$
\nabla \cdot\left(\nabla u_{1}\right)=0 \quad \text { in } \Omega, \quad \nabla \cdot\left(\nabla u_{2}\right)=0 \quad \text { in } R^{3} \backslash \bar{\Omega}
$$

transmission conditions on the interface $S$

$$
u_{1}=u_{2}, \quad \mu \frac{\partial u_{1}}{\partial n}=\frac{\partial u_{2}}{\partial n} \quad \text { on } S,
$$

and a radiation condition

$$
\lim _{|\xi| \rightarrow \infty} \nabla u_{2}=\boldsymbol{H}_{e x t}
$$

Here $\boldsymbol{H}_{i}=\nabla u_{i}, \mu=\mu_{1} / \mu_{2}$ denotes the relative permeability and $\xi=(x, y, z)$. We can express the external field in terms of the potential $u_{\text {ext }}$ as $\boldsymbol{H}_{\text {ext }}=$ $\nabla u_{e x t}$. The potential $u_{\text {ext }}$ satisfies the Laplace equation, because the Maxwell's equation $\nabla \times \boldsymbol{H}=\mathbf{0}$ is valid for nonconducting media [10,17]. A particular situation $\boldsymbol{H}_{\text {ext }}=\left(0,0, H_{0}\right)$ with the potential $u_{e x t}=H_{0} z$ is a widely-used example of the external field. Solution of the problem (2.1)-(2.3) is defined up to a constant. To fix the constant, we redefine condition (2.3) as

$$
\lim _{|\xi| \rightarrow \infty} u_{2}=u_{e x t}
$$

The same mathematical model is valid for linearly-polarizable dielectric media in an external electric field [10].

The potential $u_{1}$ satisfies the Green's representation formula

$$
u_{1}\left(\xi^{0}\right)+\int_{S}\left(\frac{\partial u^{*}}{\partial n}\left(\xi^{0}, \xi\right) u_{1}(\xi)-u^{*}\left(\xi^{0}, \xi\right) \frac{\partial u_{1}}{\partial n}(\xi)\right) d S=0, \quad \xi^{0} \in \Omega
$$

for the integration with respect to the field point $\xi$. Here $u^{*}\left(\xi^{0}, \xi\right)=1 /(4 \pi \mid \xi-$ $\left.\xi^{0} \mid\right)$ is the fundamental solution of the Laplace equation and $\boldsymbol{n}=\boldsymbol{n}(\xi)$ is the unit outward normal to $\Omega$. A potential function with the radiation conditions

$$
u(\xi)=O\left(|\xi|^{-1}\right), \quad \nabla u(\xi)=O\left(|\xi|^{-2}\right) \quad \text { for }|\xi| \rightarrow \infty
$$

satisfies the following representation formula in the unbounded domain

$$
u\left(\xi^{0}\right)-\int_{S}\left(\frac{\partial u^{*}}{\partial n}\left(\xi^{0}, \xi\right) u(\xi)-u^{*}\left(\xi^{0}, \xi\right) \frac{\partial u}{\partial n}(\xi)\right) d S=0 \quad \text { for } \xi^{0} \in R^{3} \backslash \bar{\Omega}
$$

written for the same normal vector as equation (2.5). According to condition (2.4), equation (2.6) is valid for $u=u_{2}-u_{\text {ext }}$

$u_{2}\left(\xi^{0}\right)-u_{e x t}\left(\xi^{0}\right)-\int_{S}\left(\frac{\partial u^{*}}{\partial n} u_{2}-u^{*} \frac{\partial u_{2}}{\partial n}\right) d S=-\int_{S}\left(\frac{\partial u^{*}}{\partial n} u_{e x t}-u^{*} \frac{\partial u_{e x t}}{\partial n}\right) d S$ 
for $\xi^{0} \in R^{3} \backslash \bar{\Omega}$. Integral equations (2.5) and (2.7) for $\xi^{0} \rightarrow S$, when the boundary $S$ is smooth, take the form

$$
\begin{aligned}
& \frac{1}{2} u_{1}\left(\xi^{0}\right)+\int_{S}\left(\frac{\partial u^{*}}{\partial n}\left(\xi^{0}, \xi\right) u_{1}(\xi)-u^{*}\left(\xi^{0}, \xi\right) \frac{\partial u_{1}}{\partial n}(\xi)\right) d S=0 \\
& \frac{1}{2}\left(u_{2}\left(\xi^{0}\right)-u_{e x t}\left(\xi^{0}\right)\right)-\int_{S}\left(\frac{\partial u^{*}}{\partial n}\left(\xi^{0}, \xi\right) u_{2}(\xi)-u^{*}\left(\xi^{0}, \xi\right) \frac{\partial u_{2}}{\partial n}(\xi)\right) d S \\
& \quad=-\int_{S}\left(\frac{\partial u^{*}}{\partial n}\left(\xi^{0}, \xi\right) u_{e x t}(\xi)-u^{*}\left(\xi^{0}, \xi\right) \frac{\partial u_{e x t}}{\partial n}(\xi)\right) d S=\frac{u_{e x t}\left(\xi^{0}\right)}{2}
\end{aligned}
$$

for $\xi^{0} \in S$. The last equality of equation (2.9) is due to an application of equation (2.8) to the harmonic function $u_{1}(\xi)=u_{\text {ext }}(\xi)$. Let us introduce new notations for the boundary potential $u:=u_{1}$ and the boundary flux $q:=$ $\partial u_{1} / \partial n$. We reformulate equations (2.8) and (2.9) in new notations, using transmission conditions $(2.2)$

$$
\begin{aligned}
& \frac{1}{2} u\left(\xi^{0}\right)+\int_{S}\left(\frac{\partial u^{*}}{\partial n}\left(\xi^{0}, \xi\right) u(\xi)-u^{*}\left(\xi^{0}, \xi\right) q(\xi)\right) d S=0 \\
& \frac{1}{2} u\left(\xi^{0}\right)-\int_{S}\left(\frac{\partial u^{*}}{\partial n}\left(\xi^{0}, \xi\right) u(\xi)-\mu u^{*}\left(\xi^{0}, \xi\right) q(\xi)\right) d S=u_{e x t}\left(\xi^{0}\right)
\end{aligned}
$$

for $\xi^{0} \in S$. Some manipulations with equations (2.10) and (2.11) result in the second kind boundary integral equation only for the boundary potential and the first kind boundary integral equation for the boundary flux

$$
\begin{aligned}
u\left(\xi^{0}\right)+2 \frac{\mu-1}{\mu+1} \int_{S} \frac{\partial u^{*}}{\partial n}\left(\xi^{0}, \xi\right) u(\xi) d S & =\frac{2}{\mu+1} u_{e x t}\left(\xi^{0}\right) \\
\int_{S} u^{*}\left(\xi^{0}, \xi\right) q(\xi) d S & =\frac{1}{\mu-1}\left(u_{e x t}\left(\xi^{0}\right)-u\left(\xi^{0}\right)\right)
\end{aligned}
$$

for $\xi^{0} \in S$. These equations can be solved successively to find boundary unknowns.

When the boundary $S$ is piecewise-smooth, then equations (2.8)-(2.13) are valid only on smooth parts of the surface. Integral equations (2.5) and (2.7) for $\xi^{0}$ tending to the corner point of $S$ take the form

$$
\begin{aligned}
& c\left(\xi^{0}\right) u_{1}\left(\xi^{0}\right)+\int_{S}\left(\frac{\partial u^{*}}{\partial n} u_{1}-u^{*} \frac{\partial u_{1}}{\partial n}\right) d S=0 \\
& \left(1-c\left(\xi^{0}\right)\right)\left(u_{2}\left(\xi^{0}\right)-u_{e x t}\left(\xi^{0}\right)\right)-\int_{S}\left(\frac{\partial u^{*}}{\partial n} u_{2}-u^{*} \frac{\partial u_{2}}{\partial n}\right) d S=c\left(\xi^{0}\right) u_{e x t}\left(\xi^{0}\right)
\end{aligned}
$$

for $\xi^{0} \in S$. Here the quantity $c\left(\xi^{0}\right)$ is the interior solid angle of $S$ at $\xi^{0} \in S$ and $\left(1-c\left(\xi^{0}\right)\right)$ is the outer solid angle at the same point. Both angles are divided by $4 \pi$. Analogous manipulations with boundary integral equations (2.14) and (2.15), as in the case of the smooth boundary, result in the boundary integral equation only for the boundary potential and the boundary integral equation 
for the boundary flux

$$
\begin{aligned}
& u\left(\xi^{0}\right)-2 \frac{\mu-1}{\mu+1}\left(\frac{1}{2}-c\left(\xi^{0}\right)\right) u\left(\xi^{0}\right)+2 \frac{\mu-1}{\mu+1} \\
& \times \int_{S} \frac{\partial u^{*}}{\partial n}\left(\xi^{0}, \xi\right) u(\xi) d S=\frac{2}{\mu+1} u_{e x t}\left(\xi^{0}\right), \\
& \int_{S} u^{*}\left(\xi^{0}, \xi\right) q(\xi) d S=\frac{1}{\mu-1}\left(u_{e x t}\left(\xi^{0}\right)-u\left(\xi^{0}\right)\right)
\end{aligned}
$$

for $\xi^{0} \in S$ and $S$ is piecewise-smooth. The only difference between the formulation (2.12) and (2.13) on the smooth surface and the formulation (2.16) and (2.17) on the piecewise-smooth surface is in the presence of an additional term with the solid angle in equation (2.16) for the boundary potential. The solid angle equals $1 / 2$ at every point of the smooth surface, the additional term of equation (2.16) vanishes in this case and we get equation (2.12) as a result.

The integral formulation, analogous to (2.16) and (2.17), is derived in [14]. Remark. Equations (2.13) and (2.17) are valid for $\mu \neq 1$. The case $\mu=1$ or $\mu_{1}=\mu_{2}$ corresponds to an obvious solution $u_{1}(\xi)=u_{2}(\xi)=u_{\text {ext }}(\xi)$ of the considered problem (2.1), (2.2), (2.4).

\section{Problem reformulation in a meridian plane}

An assumption about an axial symmetry of the domain $\Omega$ and of the external field $\mathbf{H}_{\text {ext }}$ allows us to reformulate boundary integral equations (2.12) and (2.13) on a meridian line $\Gamma$ of the smooth surface $S$ in cylindrical coordinates $(r, z)$. Let the boundary $\Gamma$ be described by a parametrization

$$
\Gamma=\{\xi=(r, z) \mid \quad \xi=\psi(t):=(r(t), z(t)), \quad t \in[0, L]\},
$$

such that $\left|\psi^{\prime}(t)\right|=\sqrt{\left(r^{\prime}\right)^{2}+\left(z^{\prime}\right)^{2}}>0$ for any $t \in[0, L]$.

Then boundary integral equations (2.12) and (2.13) take form

$$
\begin{aligned}
& u\left(t^{0}\right)+2 \frac{\mu-1}{\mu+1} \int_{0}^{L} a\left(t, t^{0}\right) u(t) d t=\frac{2}{\mu+1} u_{\text {ext }}\left(t^{0}\right), \\
& \int_{0}^{L} b\left(t, t^{0}\right) q(t) d t=\frac{1}{\mu-1}\left(u_{e x t}\left(t^{0}\right)-u\left(t^{0}\right)\right)
\end{aligned}
$$

for $t^{0} \in[0, L]$ with kernel functions

$$
a\left(t, t^{0}\right)=\left(\frac{\partial u_{a x}^{*}}{\partial n} \circ \psi\right)\left(t, t^{0}\right) r(t)\left|\psi^{\prime}(t)\right|, \quad b\left(t, t^{0}\right)=\left(u_{a x}^{*} \circ \psi\right)\left(t, t^{0}\right) r(t)\left|\psi^{\prime}(t)\right| .
$$

Here $\xi=(r, z), \xi^{0}=\left(r^{0}, z^{0}\right), \boldsymbol{n}=\left(n_{r}, n_{z}\right)=\left(-z^{\prime}, r^{\prime}\right) /\left|\psi^{\prime}(t)\right|$ the unit outward normal on $\Gamma$ for the clock-wise change of the parameter $t$. An axisymmetric fundamental solution $u_{a x}^{*}$ is defined in terms of the complete elliptic integral of the first kind $K(m)$, see e.g. [19],

$$
u_{a x}^{*}\left(\xi^{0}, \xi\right)=\frac{K(m)}{\pi \sqrt{\left(r+r^{0}\right)^{2}+\left(z-z^{0}\right)^{2}}},
$$




$$
K(m)=\int_{0}^{\pi / 2} \frac{d \theta}{\sqrt{1-m \sin ^{2} \theta}}, \quad m=\frac{4 r r^{0}}{\left(r+r^{0}\right)^{2}+\left(z-z^{0}\right)^{2}} .
$$

A calculation of $\partial u_{a x}^{*} / \partial n$, with the use of the relation for $K^{\prime}(m)$ in terms of the complete elliptic integral of the second kind $E(m)$, gives

$$
\begin{aligned}
\frac{\partial u_{a x}^{*}}{\partial n} & =\frac{1}{\pi \sqrt{\left(r+r^{0}\right)^{2}+\left(z-z^{0}\right)^{2}}} \\
& \times\left(\frac{n_{r}}{2 r}(E(m)-K(m))-\frac{n_{r}\left(r-r^{0}\right)+n_{z}\left(z-z^{0}\right)}{\left(r-r^{0}\right)^{2}+\left(z-z^{0}\right)^{2}} E(m)\right), \\
E(m) & =\int_{0}^{\pi / 2} \sqrt{1-m^{2} \sin ^{2} \theta} d \theta .
\end{aligned}
$$

One of the used practice for axisymmetric boundary element methods is to use polynomial approximation of the complete elliptic integrals

$$
\begin{aligned}
& K(m) \approx \tilde{K}(m)=\sum_{i=0}^{4} a_{i}(1-m)^{i}-\ln (1-m) \sum_{i=0}^{4} b_{i}(1-m)^{i} \\
& E(m) \approx \tilde{E}(m)=1+\sum_{i=1}^{4} c_{i}(1-m)^{i}-\ln (1-m) \sum_{i=1}^{4} d_{i}(1-m)^{i}
\end{aligned}
$$

for the given coefficients, see [1, page 591]. The error in these expansions is less than $2 \cdot 10^{-8}$. Approximations (3.1)-(3.2) are valid for $0 \leq m<1$. The range of variation of the parameter $m$ for the considered problem is $0 \leq m \leq 1$ and we get $m=1$ for $\xi=\xi^{0}$. To define the value of $\tilde{K}(1)$, we use the relation

$$
\lim _{m \rightarrow 1}\left[K(m)-\frac{1}{2} \ln \frac{16}{1-m}\right]=0,
$$

see [1, page 591], the value of $\tilde{E}(1)$ is defined by the fact that $E(1)=1$.

Let us denote by $\tilde{a}\left(t, t^{0}\right)$ and $\tilde{b}\left(t, t^{0}\right)$ approximations of the kernels $a\left(t, t^{0}\right)$ and $b\left(t, t^{0}\right)$, due to the handling of elliptic integrals. The numerical solution of the following boundary integral equations

$$
\begin{aligned}
& u\left(t^{0}\right)+2 \frac{\mu-1}{\mu+1} \int_{0}^{L} \tilde{a}\left(t, t^{0}\right) u(t) d t=\frac{2}{\mu+1} u_{e x t}\left(t^{0}\right), \\
& \int_{0}^{L} \tilde{b}\left(t, t^{0}\right) q(t) d t=\frac{1}{\mu-1}\left(u_{e x t}\left(t^{0}\right)-u\left(t^{0}\right)\right)
\end{aligned}
$$

for $t^{0} \in[0, L]$ with kernel functions

$$
\begin{aligned}
\tilde{a}\left(t, t^{0}\right)= & \frac{r\left|\psi^{\prime}(t)\right|}{\pi \sqrt{\left(r+r^{0}\right)^{2}+\left(z-z^{0}\right)^{2}}} \\
& \times\left(\frac{n_{r}}{2 r}(\tilde{E}(m)-\tilde{K}(m))-\frac{n_{r}\left(r-r^{0}\right)+n_{z}\left(z-z^{0}\right)}{\left(r-r^{0}\right)^{2}+\left(z-z^{0}\right)^{2}} \tilde{E}(m)\right),
\end{aligned}
$$




$$
\tilde{b}\left(t, t^{0}\right)=\frac{r\left|\psi^{\prime}(t)\right|}{\pi \sqrt{\left(r+r^{0}\right)^{2}+\left(z-z^{0}\right)^{2}}} \tilde{K}(m)
$$

for the unknown boundary potential $u$ and the boundary flux $q$ is in a focus of the present research. We find $u$ as a solution of (3.4) at the first stage, and then $q$ as a solution of (3.5) for the computed function $u$, at the second stage.

Remark. The kernels (3.6) and (3.7) are not specific for electro- and magnetostatic potential problems. Any axisymmetric potential problem in cylindrical coordinates can contain the same kernels in its integral formulation.

\subsection{Asymptotic kernels behavior}

To perform accurate integration in equations (3.4) and (3.5), we analyze an asymptotic behavior of kernel functions (3.6) and (3.7) for an integration point $t$ tending to a source point $t^{0}$.

Let us assume that only the end points of the boundary $\Gamma$ lie at the symmetry axis, i.e.

$$
r(t)=0 \quad \text { for } t \in\{0, L\}, \quad r(t) \neq 0 \quad \text { for } t \in(0, L) .
$$

The notation $t \in\{0, L\}$ means that $t$ takes values 0 or $L$. We distinguish two cases: the Case 1, when the source point does not lie at the symmetry axis $\left(t \rightarrow t^{0}, t^{0} \notin\{0, L\}\right)$, and the Case 2 , when the source point lies at the symmetry axis $\left(t \rightarrow t^{0}, t^{0} \in\{0, L\}\right)$.

\subsubsection{Case 1. Source point is not at the symmetry axis}

We have that $m=4 r r^{0} /\left(\left(r+r^{0}\right)^{2}+\left(z-z^{0}\right)^{2}\right) \rightarrow 1$ in the Case 1. Kernel functions (3.6) and (3.7) contain the term $\tilde{K}(m)$ at their formulations. According to the limit value (3.3), $\tilde{K}(m)$ has a logarithmic singularity at $m=1$, hence the integrals in (3.4) and (3.5) are logarithmically singular in the Case 1.

\subsubsection{Case 2. Source point is at the symmetry axis}

We have that $m=0$ for $t^{0} \in\{0, L\}$ and $t \neq t_{0}$. It allows us to redefine the kernel functions (3.6) and (3.7) for $t^{0} \in\{0, L\}$ by setting $m=0$ for all integration points $t$

$$
\begin{aligned}
& \tilde{a}\left(t, t^{0}\right):=\frac{r\left|\psi^{\prime}(t)\right|}{\pi \sqrt{\left(r+r^{0}\right)^{2}+\left(z-z^{0}\right)^{2}}} \\
& \times\left(\frac{n_{r}}{2 r}(\tilde{E}(0)-\tilde{K}(0))-\frac{n_{r}\left(r-r^{0}\right)+n_{z}\left(z-z^{0}\right)}{\left(r-r^{0}\right)^{2}+\left(z-z^{0}\right)^{2}} \tilde{E}(0)\right), \\
& \tilde{b}\left(t, t^{0}\right):=\frac{r\left|\psi^{\prime}(t)\right|}{\pi \sqrt{\left(r+r^{0}\right)^{2}+\left(z-z^{0}\right)^{2}}} \tilde{K}(0), \quad t^{0} \in\{0, L\} .
\end{aligned}
$$


For a further simplification of the kernels, we use the exact values of the elliptic integrals at $m=0$, namely, $K(0)=\pi / 2$ and $E(0)=\pi / 2$, instead of their approximations $\tilde{K}(0)$ and $\tilde{E}(0)$. It gives that

$$
\begin{aligned}
& \tilde{a}\left(t, t^{0}\right)=-\frac{r\left|\psi^{\prime}(t)\right|}{2 \sqrt{\left(r+r^{0}\right)^{2}+\left(z-z^{0}\right)^{2}}} \frac{n_{r}\left(r-r^{0}\right)+n_{z}\left(z-z^{0}\right)}{\left(r-r^{0}\right)^{2}+\left(z-z^{0}\right)^{2}}, \\
& \tilde{b}\left(t, t^{0}\right)=\frac{r\left|\psi^{\prime}(t)\right|}{2 \sqrt{\left(r+r^{0}\right)^{2}+\left(z-z^{0}\right)^{2}}}, \quad t^{0} \in\{0, L\} .
\end{aligned}
$$

We get in the limit that

$$
\lim _{\text {Case } 2} \frac{r}{\sqrt{\left(r+r^{0}\right)^{2}+\left(z-z^{0}\right)^{2}}}=\frac{1}{2},
$$

hence

$$
\begin{aligned}
\lim _{\text {Case } 2} \tilde{a}\left(t, t^{0}\right) & =-\frac{1}{4} \lim _{\text {Case } 2} \frac{-z^{\prime}\left(r-r^{0}\right)+r^{\prime}\left(z-z^{0}\right)}{\left(r-r^{0}\right)^{2}+\left(z-z^{0}\right)^{2}}, \\
\lim _{\text {Case } 2} \tilde{b}\left(t, t^{0}\right) & =\frac{1}{4}\left|\psi^{\prime}\left(t^{0}\right)\right|
\end{aligned}
$$

for $n_{r}=-z^{\prime}(t) /\left|\psi^{\prime}(t)\right|$ and $n_{z}=r^{\prime}(t) /\left|\psi^{\prime}(t)\right|$. Let us distinguish two situations, when $n_{r}=0$ at the symmetry axis and $n_{r} \neq 0$. If $n_{r}=0$, then the kernel (3.10) in the limit is the indeterminate form $0 / 0$. We apply L'Hopital's rule to evaluate the limit and get convergence. If $n_{r} \neq 0$, the kernel (3.10) diverges to infinity in the Case 2, i.e. has an algebraic singularity.

We summarize that kernels (3.6) and (3.7) are logarithmically singular in the Case 1. Kernel (3.6), modified by (3.10) for the source point at the symmetry axis, has an algebraic singularity in the Case 2 , when $n_{r} \neq 0$ at the symmetry axis, otherwise, it is a smooth function. Kernel (3.7), modified by (3.11) for the source point at the symmetry axis, is smooth in the Case 2.

\subsection{Kernels on a circular arc}

We consider a particular case of the meridian line $\Gamma$ as a circular arc of the angle $180^{\circ}$ and the radius 1 , which rotation around $z$-axis produces a sphere centered at the origin

$$
\Gamma_{0}=\{(r, z) \mid \quad r(t)=\sin t, z(t)=\cos t, \quad t \in[0, \pi]\} .
$$

The corresponding outward unit normal vector is

$$
\boldsymbol{n}=\left(-z^{\prime}, r^{\prime}\right) / \sqrt{\left(r^{\prime}\right)^{2}+\left(z^{\prime}\right)^{2}}=(\sin t, \cos t) .
$$

After some simplifications the kernel functions take the form

$$
\begin{aligned}
\tilde{a}_{0}\left(t, t^{0}\right):=\left.\tilde{a}\left(t, t^{0}\right)\right|_{\Gamma_{0}} & =\frac{\sin t}{\pi \sqrt{2-2 \cos \left(t+t^{0}\right)}}\left(\frac{1}{2}(\tilde{E}(m)-\tilde{K}(m))-\frac{1}{2} \tilde{E}(m)\right) \\
& =-\frac{1}{2} \frac{\sin t}{\pi \sqrt{2-2 \cos \left(t+t^{0}\right)}} \tilde{K}(m),
\end{aligned}
$$




$$
\tilde{b}_{0}\left(t, t^{0}\right):=\left.\tilde{b}\left(t, t^{0}\right)\right|_{\Gamma_{0}}=\frac{\sin t}{\pi \sqrt{2-2 \cos \left(t+t^{0}\right)}} \tilde{K}(m)=-2 \tilde{a}_{0}\left(t, t^{0}\right),
$$

where

$$
m=\frac{2 \sin t \sin t^{0}}{1-\cos \left(t+t^{0}\right)}, \quad t, t^{0} \in[0, \pi] .
$$

The redefined kernels (3.10) and (3.11) take the following form

$$
\begin{aligned}
& \tilde{a}_{0}\left(t, t^{0}\right)=-\frac{1}{4} \frac{\sin t}{\sqrt{2-2 \cos \left(t+t^{0}\right)}}, \quad \tilde{b}_{0}\left(t, t^{0}\right)=-2 \tilde{a}_{0}(t,\{0, \pi\}), \quad t^{0} \in\{0, \pi\}, \\
& \lim _{\text {Case } 2} \tilde{a}_{0}\left(t, t^{0}\right)=-\frac{1}{8}, \quad \lim _{\text {Case } 2} \tilde{b}_{0}\left(t, t^{0}\right)=\frac{1}{4} .
\end{aligned}
$$

\section{Collocation on polygonal boundaries}

Let

$$
T_{n}=\left\{t_{i} \mid \quad 0=t_{0}<t_{1}<\ldots<t_{n}=L, \quad i=\overline{0, n}\right\}
$$

be a partition of the parameter interval $[0, L]$. Let the parametrization $\psi(t)$ of $\Gamma$ be interpolated by piecewise-linear functions $\psi_{h}(t)=\left(r_{h}(t), z_{h}(t)\right)$, subject to the partition $T_{n}$,

$$
\Gamma_{h}=\left\{\xi \mid \xi=\psi_{h}(t)=\xi_{j-1}+\frac{t-t_{j-1}}{t_{j}-t_{j-1}}\left(\xi_{j}-\xi_{j-1}\right), t \in\left[t_{j-1}, t_{j}\right], j=\overline{1, n}\right\} .
$$

The kernel functions on $\Gamma_{h}$ are defined from (3.6) and (3.7) as follows

$$
\begin{aligned}
& a_{h}\left(t, t^{0}\right)=\frac{r_{h}\left|\psi_{h}^{\prime}(t)\right|}{\pi \sqrt{\left(r_{h}+r_{h}^{0}\right)^{2}+\left(z_{h}-z_{h}^{0}\right)^{2}}} \times \\
&\left(\frac{\left(n_{h}\right)_{r}}{2 r_{h}}\left(\tilde{E}_{h}\left(m_{h}\right)-\tilde{K}_{h}\left(m_{h}\right)\right)-\frac{\left(n_{h}\right)_{r}\left(r_{h}-r_{h}^{0}\right)+\left(n_{h}\right)_{z}\left(z_{h}-z_{h}^{0}\right)}{\left(r_{h}-r_{h}^{0}\right)^{2}+\left(z_{h}-z_{h}^{0}\right)^{2}} \tilde{E}_{h}\left(m_{h}\right)\right), \\
& b_{h}\left(t, t^{0}\right)=\frac{r_{h}\left|\psi_{h}^{\prime}(t)\right|}{\pi \sqrt{\left(r_{h}+r_{h}^{0}\right)^{2}+\left(z_{h}-z_{h}^{0}\right)^{2}}} \tilde{K}_{h}\left(m_{h}\right)
\end{aligned}
$$

with the modification of the kernels for the collocation at the end points, due to $(3.10)$ and $(3.11)$,

$$
\begin{aligned}
& a_{h}\left(t, t^{0}\right)=-\frac{r_{h}\left|\psi_{h}^{\prime}(t)\right|}{2 \sqrt{\left(r_{h}+r_{h}^{0}\right)^{2}+\left(z_{h}-z_{h}^{0}\right)^{2}}} \frac{\left(n_{h}\right)_{r}\left(r_{h}-r_{h}^{0}\right)+\left(n_{h}\right)_{z}\left(z_{h}-z_{h}^{0}\right)}{\left(r_{h}-r_{h}^{0}\right)^{2}+\left(z_{h}-z_{h}^{0}\right)^{2}}, \\
& b_{h}\left(t, t^{0}\right)=\frac{r_{h}\left|\psi_{h}^{\prime}(t)\right|}{2 \sqrt{\left(r_{h}+r_{h}^{0}\right)^{2}+\left(z_{h}-z_{h}^{0}\right)^{2}}}, \quad t^{0} \in\{0, L\} .
\end{aligned}
$$

Here the unit outward normal (for the clock-wise change of the parameter $t$ ) is piecewise-constant at the element

$$
\boldsymbol{n}_{h}=\left(-\frac{z_{j}-z_{j-1}}{t_{j}-t_{j-1}}, \frac{r_{j}-r_{j-1}}{t_{j}-t_{j-1}}\right) / \sqrt{\frac{\left(r_{j}-r_{j-1}\right)^{2}+\left(z_{j}-z_{j-1}\right)^{2}}{\left(t_{j}-t_{j-1}\right)^{2}}}, t \in\left[t_{j-1}, t_{j}\right]
$$


and

$$
m_{h}=\frac{4 r_{h} r_{h}^{0}}{\left(r_{h}+r_{h}^{0}\right)^{2}+\left(z_{h}-z_{h}^{0}\right)^{2}}, \quad \xi^{0}=\left(r_{h}^{0}, z_{h}^{0}\right)=\xi_{j-1}+\frac{t^{0}-t_{j-1}}{t_{j}-t_{j-1}}\left(\xi_{j}-\xi_{j-1}\right) .
$$

Let $S_{k}\left(T_{n}\right)$ denotes the polynomial B-splines spaces of order $k$, subject to the partition $T_{n}$

$S_{k}\left(T_{n}\right)= \begin{cases}\left\{u \in L^{2}(0, L)|u|_{\left(t_{j-1}, t_{j}\right)} \in P_{0}\left(t_{j-1}, t_{j}\right), j=\overline{1, n}\right\} & \text { for } k=0, \\ \left\{u \in C^{k-1}(0, L)|u|_{\left(t_{j-1}, t_{j}\right)} \in P_{k}\left(t_{j-1}, t_{j}\right), j=\overline{1, n}\right\} & \text { for } k \geq 1 .\end{cases}$

We use the notation $P_{0}\left(T_{n}\right)=S_{0}\left(T_{n}\right)$ and $P_{1}\left(T_{n}\right)=S_{1}\left(T_{n}\right)$ for the piecewiseconstant and the piecewise-linear spaces. The collocation points are defined over the parameter set $T_{n}^{0}$

$$
\begin{array}{lrl}
T_{n}^{0}=\left\{t_{i}^{0} \mid \quad t_{i}^{0}=\left(t_{i-1}+t_{i}\right) / 2,\right. & i=\overline{1, n}\} & \text { for } k \text { even, } \\
T_{n}^{0}=T_{n} & \text { for } k \text { odd }
\end{array}
$$

as midpoints for $k$ even and nodal points for $k$ odd, see e.g. [3].

The equal order $S_{k}$-collocation of equations (3.4) and (3.5), modified on the piecewise-smooth surface according to (2.16) and (2.17), is to find $\left(u_{h}, q_{h}\right) \in$ $S_{k}\left(T_{n}\right) \times S_{k}\left(T_{n}\right)$ such that for $t^{0} \in T_{n}^{0}$

$$
\begin{aligned}
& u_{h}\left(t^{0}\right)+2 \frac{\mu-1}{\mu+1}\left(\left(\frac{1}{2}-c\left(t^{0}\right)\right) u_{h}\left(t^{0}\right)+\int_{0}^{L} a_{h}\left(t, t^{0}\right) u_{h}(t) d t\right)=\frac{2}{\mu+1} u_{e x t}\left(t^{0}\right) \\
& \int_{0}^{L} b_{h}\left(t, t^{0}\right) q_{h}(t) d t=\frac{1}{\mu-1}\left(u_{e x t}\left(t^{0}\right)-u_{h}\left(t^{0}\right)\right) .
\end{aligned}
$$

A simplification of the kernel function $a_{h}\left(t, t^{0}\right)$ is possible, when the field and the collocation points lie at the same boundary element, i.e. $t$ and $t^{0} \in$ $\left[t_{j-1}, t_{j}\right]$. The factor $n_{r}\left(r_{h}-r_{h}^{0}\right)+n_{z}\left(z_{h}-z_{h}^{0}\right)$ vanishes in this case and

$$
a_{h}\left(t, t^{0}\right)=\frac{\sqrt{\left(r_{h}^{\prime}\right)^{2}+\left(z_{h}^{\prime}\right)^{2}}}{\pi \sqrt{\left(r_{h}+r_{h}^{0}\right)^{2}+\left(z_{h}-z_{h}^{0}\right)^{2}}} \frac{n_{r}}{2}\left(\tilde{E}\left(m_{h}\right)-\tilde{K}\left(m_{h}\right)\right)
$$

for $t$ and $t^{0} \in\left[t_{j-1}, t_{j}\right]$. The simplification (4.5) changes an asymptotic behavior of the kernel $a_{h}\left(t, t^{0}\right)$ in the Case 2, when the collocation point lies at the symmetry axis $\left(t \rightarrow t^{0}, t^{0} \in\{0, L\}\right)$. As it was shown in Section 3.1, we have that the kernel $\tilde{a}\left(t, t^{0}\right)$ has an algebraic singularity in the Case 2 , when $n_{r} \neq 0$ at the symmetry axis. We do have a situation $n_{r} \neq 0$ at the symmetry axis for polygonal boundaries, but this singularity is suppressed by vanishing of the factor $n_{r}\left(r_{h}-r_{h}^{0}\right)+n_{z}\left(z_{h}-z_{h}^{0}\right)$. Moreover, according to the modification (4.1), we get that

$$
a_{h}(t, 0)=0 \text { for } t \in\left[t_{0}, t_{1}\right] \text { and } a_{h}(t, L)=0 \text { for } t \in\left[t_{n-1}, t_{n}\right] .
$$

We summarize that kernels $a_{h}\left(t, t^{0}\right)$ and $b_{h}\left(t, t^{0}\right)$ are logarithmically singular in the Case 1 and smooth in the Case 2.

Remark. Midpoint collocations include only the Case $1\left(t \rightarrow t^{0}, t^{0} \notin\{0, L\}\right)$, whereas nodal collocations contain both the Case 1 and the Case $2\left(t \rightarrow t^{0}, t^{0} \in\right.$ $\{0, L\})$. 


\section{Test computations}

\section{$5.1 \quad$ Test 1}

We perform test computations to compare the accuracy and the empirical order of convergence (eoc) of the axisymmetric collocation method (4.3)-(4.4) on the polygonal interpolation $\Gamma_{h}$ of the circular arc $\Gamma_{0}$. We compute the solution of the collocation problem for equal-order approximations of the boundary potential $u_{h}$ and the boundary flux $q_{h}$ on uniformly refined meshes. The initial mesh (level 0) consists of 8 boundary elements, the computations are performed up to the level 6 with 512 elements. The approximate solutions of the piecewiseconstant midpoint collocation $\left(P_{0}\right.$-collocation $)$ and the piecewise-linear nodal collocation $\left(P_{1}\right.$-collocation) on the boundary $\Gamma_{h}$ are compared with an analytically known solution on $\Gamma_{0}$.

The original exterior transmission problem (2.1), (2.2), (2.4) for a uniform external field $\boldsymbol{H}_{\text {ext }}=\left(0,0, H_{0}\right)$ was studied for a dielectric ellipsoid in [10] and for a magnetic-fluid sphere in [17]. A solution was sought in spherical coordinates by the method of separation of variables. An exact solution for a unit sphere is

$$
\begin{aligned}
& u_{1}(x, y, z)=\frac{3 H_{0}}{\mu+2} z, \quad \text { in } \Omega, \\
& u_{2}(x, y, z)=H_{0} z+\frac{\mu-1}{\mu+2} H_{0} \frac{z}{\left(x^{2}+y^{2}+z^{2}\right)^{3 / 2}}, \quad \text { in } R^{3} \backslash \bar{\Omega} .
\end{aligned}
$$

The corresponding boundary potential $u$ and the boundary flux $q$ on the meridian line are

$$
u(r, z)=\frac{3 H_{0}}{\mu+2} z, \quad q(r, z)=\frac{\partial u}{\partial r} \sin t+\frac{\partial u}{\partial z} \cos t=\frac{3 H_{0}}{\mu+2} z \quad(r, z) \in \Gamma_{0} .
$$

We set the radius of the circular arc equals one, $\mu=6$ and $H_{0}=1$ for computations. The computations in this paper were performed by using Maple ${ }^{\mathrm{TM}}[4]$.

The solid angle is computed, using the identity

$$
c\left(\xi^{0}\right)=-\int_{S} \frac{\partial u^{*}}{\partial n}\left(\xi^{0}, \xi\right) d S, \quad \xi^{0} \in S .
$$

The approximate value of the solid angle is obtained from the collocation of equation (4.3) over $T_{n}^{0}$ for $c\left(t^{0}\right)=1 / 2$. Namely, to get the integral in the right-hand side of (5.1), we sum the row elements of the corresponding matrix, subtract one (this coefficient is die to the first term of (4.3)) and divide by the quantity $2(\mu-1) /(\mu+1)$. When the solid angle is computed, the diagonal matrix elements are modified, according to the second term of equation (4.3). The approximate solid angle is used only for the $P_{1}$-collocation on the polygonal boundary, the $P_{0}$-collocation and the $P_{1}$-collocation on the smooth boundary are computed with the angle equals $1 / 2$.

We perform the numerical evaluation of all integrals arising in the method. Namely, we employ the Gaussian integration with 12 points at the boundary 
element for the numerical approximation of regular integrals and the logarithmically weighted Gaussian formula with 8 points for weakly singular integrals. 12 points for regular integrals is an overestimated value, which was specially taken for computations to exclude an integration error from the complete error of the collocation solution. The number of integration points for regular integrals depends on the distance between the collocation point and the element being integrated. We have performed test computations on the circular arc and on its polygonal approximation at the different mesh levels of the Test 1 to get the relative error of about $10^{-10}$ if to compare with the 12 points Gaussian integration. The near-weakly-singular integrals require 6 points on the smooth boundary and 8 points on the polygonal boundary, whereas the regular integrals far from singularities can be computed with 4 points. We have analogous results for both kernels at the every mesh level. The near-singular integrals for the collocation at the symmetry axis have appeared to be more sensitive to the kernel function and the boundary representation. Namely, they require 4 points on the smooth boundary for both kernels, 8 points on the polygonal boundary for the kernel $a_{h}\left(t, t^{0}\right)$ and 4 points on the polygonal boundary for the kernel $b_{h}\left(t, t^{0}\right)$. We have found that 8 points for the weakly-singular integrals should be sufficient for numerical integration by the logarithmically weighted Gaussian formula. The relative error, if to compare with the 12 points integration, is about $10^{-8}$ at the near-axis elements for the near-axis collocation and has the machine precision $10^{-15}$ for the remaining cases. The error behavior indicates the nearness of the sharp kernels variation close to the symmetry axis. We note that a rearrangement of the kernels is required for the application of the logarithmically weighted Gaussian integration. Some details can be found in [5] for axisymmetric potential problems.

The observed properties of the integrals for the collocation at the symmetry axis supports the analytical results of Section 3.1 and Section 4 about the kernels behavior near the symmetry axis. Strongly singular integrals are absent in the formulation on smooth boundaries, due to the property $n_{r}=0$ at the symmetry axis, and only 4 integration points are necessary for accurate integration of the near-singular integrals for the axis collocation. Strongly singular integrals are present in the formulation on the polygonal boundaries for the kernel $a_{h}\left(t, t^{0}\right)$ but they are suppressed at the symmetry axis, due to the kernel simplification (4.5). That is why we need 8 points for the near-singular integrals at the axis collocation of the polygonal boundary.

The notations $E(\infty, u)$ and $E(2, u)$ are used for errors in the $L^{\infty}$ - and the $L_{r}^{2}$-norms, respectively,

$$
\begin{aligned}
& E(\infty, u):=\max _{t^{0} \in T_{n}^{0}}\left|u\left(t^{0}\right)-u_{h}\left(t^{0}\right)\right|, \quad E(2, u):=L_{r}^{2}\left(u-u_{h}\right), \\
& L_{r}^{2}(u):=\left(\int_{0}^{L}|u(t)|^{2} r(t)\left|\psi^{\prime}(t)\right| d t\right)^{1 / 2} .
\end{aligned}
$$

The $L^{\infty}$-norm is computed at the collocation points. We use the midpoint rule for the numerical evaluation of the $L_{r}^{2}$-norm of the $P_{0}$-collocation error, because the computations are performed only at the collocation points in this case. We use the Gaussian integration with 6 points for the numerical evaluation of the 
$L_{r}^{2}$-norm for the $P_{1}$-collocation error on the smooth boundary and the 2 points rule on the polygonal boundary. The relative error, if to compare with the 12 points integration, has the machine precision $10^{-15}$ at the every mesh level.

Table 1. Errors and orders of convergence of the $P_{0}$-collocation on the circular arc

\begin{tabular}{lllllllll}
\hline$n$ & $E(\infty, u)$ & eoc & $E(2, u)$ & eoc & $E(\infty, q)$ & eoc & $E(2, q)$ & eoc \\
\hline 8 & $.477 \mathrm{e}-3$ & & $.305 \mathrm{e}-3$ & & $.178 \mathrm{e}-2$ & & $.118 \mathrm{e}-2$ & \\
16 & $.155 \mathrm{e}-3$ & 1.625 & $.936 \mathrm{e}-4$ & 1.704 & $.601 \mathrm{e}-3$ & 1.568 & $.357 \mathrm{e}-3$ & 1.719 \\
32 & $.432 \mathrm{e}-4$ & 1.840 & $.254 \mathrm{e}-4$ & 1.884 & $.167 \mathrm{e}-3$ & 1.851 & $.964 \mathrm{e}-4$ & 1.888 \\
64 & $.113 \mathrm{e}-4$ & 1.932 & $.657 \mathrm{e}-5$ & 1.948 & $.435 \mathrm{e}-4$ & 1.939 & $.250 \mathrm{e}-4$ & 1.949 \\
128 & $.289 \mathrm{e}-5$ & 1.970 & $.167 \mathrm{e}-5$ & 1.976 & $.111 \mathrm{e}-4$ & 1.966 & $.635 \mathrm{e}-5$ & 1.976 \\
256 & $.729 \mathrm{e}-6$ & 1.985 & $.421 \mathrm{e}-6$ & 1.988 & $.282 \mathrm{e}-5$ & 1.979 & $.160 \mathrm{e}-5$ & 1.988 \\
512 & $.183 \mathrm{e}-6$ & 1.991 & $.106 \mathrm{e}-6$ & 1.994 & $.751 \mathrm{e}-6$ & 1.910 & $.402 \mathrm{e}-6$ & 1.994 \\
\hline
\end{tabular}

Table 2. Errors and orders of convergence of the $P_{0}$-collocation on the polygonal boundary

\begin{tabular}{lllllllll}
\hline$n$ & $E(\infty, u)$ & eoc & $E(2, u)$ & eoc & $E(\infty, q)$ & eoc & $E(2, q)$ & eoc \\
\hline 8 & $.158 \mathrm{e}-1$ & & $.107 \mathrm{e}-1$ & & $.286 \mathrm{e}-2$ & & $.191 \mathrm{e}-2$ & \\
16 & $.414 \mathrm{e}-2$ & 1.933 & $.276 \mathrm{e}-2$ & 1.954 & $.829 \mathrm{e}-3$ & 1.784 & $.494 \mathrm{e}-3$ & 1.951 \\
32 & $.105 \mathrm{e}-2$ & 1.977 & $.698 \mathrm{e}-3$ & 1.984 & $.222 \mathrm{e}-3$ & 1.901 & $.125 \mathrm{e}-3$ & 1.987 \\
64 & $.265 \mathrm{e}-3$ & 1.991 & $.175 \mathrm{e}-3$ & 1.994 & $.573 \mathrm{e}-4$ & 1.953 & $.312 \mathrm{e}-4$ & 1.997 \\
128 & $.663 \mathrm{e}-4$ & 1.996 & $.439 \mathrm{e}-4$ & 1.997 & $.146 \mathrm{e}-4$ & 1.977 & $.782 \mathrm{e}-5$ & 1.999 \\
256 & $.166 \mathrm{e}-4$ & 1.998 & $.110 \mathrm{e}-4$ & 1.999 & $.367 \mathrm{e}-5$ & 1.989 & $.195 \mathrm{e}-5$ & 2.000 \\
512 & $.415 \mathrm{e}-5$ & 1.999 & $.275 \mathrm{e}-5$ & 1.999 & $.921 \mathrm{e}-6$ & 1.994 & $.489 \mathrm{e}-6$ & 2.000 \\
\hline
\end{tabular}

Table 1 and Table 2 illustrate the numerical convergence of the axisymmetric $P_{0}$-collocation method on the circular arc and on its polygonal interpolation, respectively. The first observation is that the polygonal boundary interpolation leads to a reduction of the computational accuracy but only for the boundary potential. In contrast to integral equation (3.5) for the boundary flux, equation (3.4) contains the normal vector in the kernel function, which is a piecewiseconstant function on the interpolated boundary. It might be a reason for the reduction of the accuracy in the computations of the boundary potential from equation (3.4). The second observation is that the boundary interpolation by the polygonal curve does not change the convergence order of the $P_{0}$-collocation method. We get the second order for the boundary potential and the boundary flux both in the $L^{\infty}$ - and the $L_{r}^{2}$-norms. We note that the $L^{\infty}$-norm of the error for the $P_{0}$-collocation on the smooth and the polygonal surfaces falls to one, if the norm is computed by the Gaussian integration (the results in Tables 1 and 2 are computed by the midpoint rule).

Table 3 and Table 4 illustrate the numerical convergence of the axisymmetric $P_{1}$-collocation method on the circular arc and on its polygonal interpolation, respectively. The boundary interpolation by the polygonal curve leads to a reduction of the computational accuracy at the collocation points both for the boundary potential and the boundary flux. Another observation is that the boundary interpolation does not change the convergence order of the 
Table 3. Errors and orders of convergence of the $P_{1}$-collocation on the circular arc

\begin{tabular}{lllllllll}
\hline$n$ & $E(\infty, u)$ & eoc & $E(2, u)$ & eoc & $E(\infty, q)$ & eoc & $E(2, q)$ & eoc \\
\hline 8 & $.147 \mathrm{e}-2$ & & $.380 \mathrm{e}-2$ & & $.711 \mathrm{e}-2$ & & $.128 \mathrm{e}-2$ & \\
16 & $.373 \mathrm{e}-3$ & 1.982 & $.957 \mathrm{e}-3$ & 1.990 & $.181 \mathrm{e}-2$ & 1.972 & $.328 \mathrm{e}-3$ & 1.968 \\
32 & $.937 \mathrm{e}-4$ & 1.993 & $.240 \mathrm{e}-3$ & 1.995 & $.458 \mathrm{e}-3$ & 1.984 & $.830 \mathrm{e}-4$ & 1.981 \\
64 & $.235 \mathrm{e}-4$ & 1.997 & $.602 \mathrm{e}-4$ & 1.997 & $.115 \mathrm{e}-3$ & 1.992 & $.209 \mathrm{e}-4$ & 1.989 \\
128 & $.588 \mathrm{e}-5$ & 1.998 & $.151 \mathrm{e}-4$ & 1.999 & $.288 \mathrm{e}-4$ & 1.999 & $.524 \mathrm{e}-5$ & 1.994 \\
256 & $.147 \mathrm{e}-5$ & 1.999 & $.377 \mathrm{e}-5$ & 1.999 & $.734 \mathrm{e}-5$ & 1.971 & $.131 \mathrm{e}-5$ & 1.997 \\
512 & $.368 \mathrm{e}-6$ & 2.000 & $.942 \mathrm{e}-6$ & 2.000 & $.248 \mathrm{e}-5$ & 1.564 & $.329 \mathrm{e}-6$ & 1.998 \\
\hline
\end{tabular}

Table 4. Errors and orders of convergence of the $P_{1}$-collocation on the polygonal boundary

\begin{tabular}{lllllllll}
\hline$n$ & $E(\infty, u)$ & eoc & $E(2, u)$ & eoc & $E(\infty, q)$ & eoc & $E(2, q)$ & eoc \\
\hline 8 & $.544 \mathrm{e}-2$ & & $.290 \mathrm{e}-2$ & & $.137 \mathrm{e}-1$ & & $.376 \mathrm{e}-2$ & \\
16 & $.124 \mathrm{e}-2$ & 2.130 & $.693 \mathrm{e}-3$ & 2.068 & $.347 \mathrm{e}-2$ & 1.979 & $.965 \mathrm{e}-3$ & 1.962 \\
32 & $.296 \mathrm{e}-3$ & 2.067 & $.168 \mathrm{e}-3$ & 2.041 & $.875 \mathrm{e}-3$ & 1.987 & $.245 \mathrm{e}-3$ & 1.976 \\
64 & $.723 \mathrm{e}-4$ & 2.034 & $.414 \mathrm{e}-4$ & 2.022 & $.220 \mathrm{e}-3$ & 1.993 & $.619 \mathrm{e}-4$ & 1.987 \\
128 & $.179 \mathrm{e}-4$ & 2.017 & $.103 \mathrm{e}-4$ & 2.012 & $.550 \mathrm{e}-4$ & 1.999 & $.155 \mathrm{e}-4$ & 1.993 \\
256 & $.444 \mathrm{e}-5$ & 2.009 & $.256 \mathrm{e}-5$ & 2.006 & $.138 \mathrm{e}-4$ & 1.994 & $.390 \mathrm{e}-5$ & 1.997 \\
512 & $.111 \mathrm{e}-5$ & 2.004 & $.638 \mathrm{e}-6$ & 2.003 & $.391 \mathrm{e}-5$ & 1.822 & $.975 \mathrm{e}-6$ & 2.001 \\
\hline
\end{tabular}

$P_{1}$-collocation. We get the second order for the boundary potential and the boundary flux both in the $L^{\infty}$ - and the $L_{r}^{2}$-norms. The numerical results in Table 4 are computed taking into account the approximate solid angle at the corner collocations. If we define the solid angle equals $1 / 2$ then the computational accuracy of the boundary potential reduces and the convergence order falls to one (this results are not presented in Table 4).

Let us compare the numerical results of Tables 1-4 with the existing theory in $2 \mathrm{D}$ for smooth boundaries $[3,9,16]$ and polygonal boundaries $[7,8]$. The axisymmetric $P_{0}$-collocation shows a superconvergence with the second order in the $L^{\infty}$-norm both on the smooth boundary and on its interpolation, see Table 1 and Table 2. The same convergence was predicted in [9, Theorem 4.6.17] for spline collocations of order 0 on two-dimensional smooth geometries. The axisymmetric $P_{1}$-collocation on the smooth boundary converges with the second order in the $L^{\infty}$-norm, see Table 3 . The same convergence was predicted in [16] for spline collocations of odd order on two-dimensional smooth geometries. The theory for the collocation in 2D on smooth boundaries [3] and on polygonal boundaries for the first kind boundary integral equations [8] predicts that the convergence in the $L^{2}$-norm (the norm is weighted on polygons) equals $k+1$ for the collocation method of order $k$. The axisymmetric boundary unknowns on the circular arc and on the polygonal interpolation show for the $P_{0}$-collocation a one order higher convergence in the $L_{r}^{2}$-norm than predicted theoretically in [3], whereas the convergence of the $P_{1}$-collocation coincides with the theory. The second order convergence in the $L_{r}^{2}$-norm for the $P_{0}$-collocation is due to the use of only collocation points for the error evaluation by the midpoint rule. If the Gaussian integration is applied then the convergence order falls to one 
and the results coincides with the theoretical predictions in [3]. It is important to note that the $P_{1}$-collocation on the polygonal boundary requires to take into consideration the solid angle at the corner points to get the theoretically predicted orders in the $L^{\infty}$ - and the $L_{r}^{2}$-norms. If the solid angle is defined as in the smooth case $c\left(\xi^{0}\right)=1 / 2$, then the convergence order reduces by one. According to the comparison of the numerical results for the axisymmetric collocation with theoretical results in $[3,7,8,9,16]$ for $2 \mathrm{D}$-collocation on smooth and polygonal boundaries, we have got that the axisymmetric collocation on the circular arc and on the polygonal interpolation shows the same convergence as $2 \mathrm{D}$-collocation. The $L_{r}^{2}$-error for the $P_{0}$-collocation, computed only at the collocation points by the midpoint rule, shows a one order higher convergence than the theory predicts. The $P_{1}$-collocation on the polygonal boundary shows a decrease of the convergence order by one if the solid angle is not included into the integral formulation.

\section{$5.2 \quad$ Test 2}

We perform test computations of the boundary field in a uniform external field $\boldsymbol{H}_{\text {ext }}=\left(0,0, H_{0}\right)$ for the different values of the relative permeability $\mu$. We consider the axisymmetric $P_{0^{-}}$and $P_{1}$-collocation method on the circular arc $\Gamma_{0}$ and its polygonal interpolation $\Gamma_{h}$. The approximate boundary field

$$
\boldsymbol{H}_{h}=\frac{\partial \phi_{h}}{\partial t} \boldsymbol{t}+q_{h} \boldsymbol{n}
$$

is evaluated at the centers of the boundary elements for the $P_{0}$-collocation and at the corners for the $P_{1}$-collocation. The difference approximation is used to compute the tangential derivative of the potential. We compare the approximate boundary field $\boldsymbol{H}_{h}$ with an analytically known solution $\boldsymbol{H}=$ $\left(0, \frac{3 H_{0}}{\mu+2}\right)$ on $\Gamma_{0}$, see Section 5.1. We compute the solution of the collocation problem on the uniform mesh with $n=12$ boundary elements on the circular arc of radius one. We set $H_{0}=17$ and $n=12$ for computations to get numerical results, comparable with those in the example on a sphere in [2].

Figure 2 presents the dependence of the relative $L_{r}^{2}$-error for the boundary field on the relative permeability $\mu$ for different variants of the collocation method. The $L_{r}^{2}$-norm is computed with the 6 point Gaussian rule. Figure 2 shows that the error tends to a constant value for increasing $\mu$. The computations with 30 digits for floating-points numbers are performed up to $\mu=10^{50}$ without loss in accuracy for increasing $\mu$. However, the error starts increasing about $\mu=10^{8}$ for the computations with 15 digits. We get that our computations with 30 digits for floating-points show a better stability in accuracy than the results with 15 digits. Comparing the accuracy of different variants of the method, we get that the $P_{0}$-collocation is slightly more accurate than the $P_{1}$-collocation, and the error, due to the boundary approximation, is more pronounced for the $P_{0}$-collocation than for the $P_{1}$-collocation. To some extent, we can compare the accuracy of the $P_{1}$-collocation on the polygonal boundary $\Gamma_{h}$ with three-dimensional computations in [2]. We get the relative error similar to the double layer potential formulation with the rewritten evaluation in 


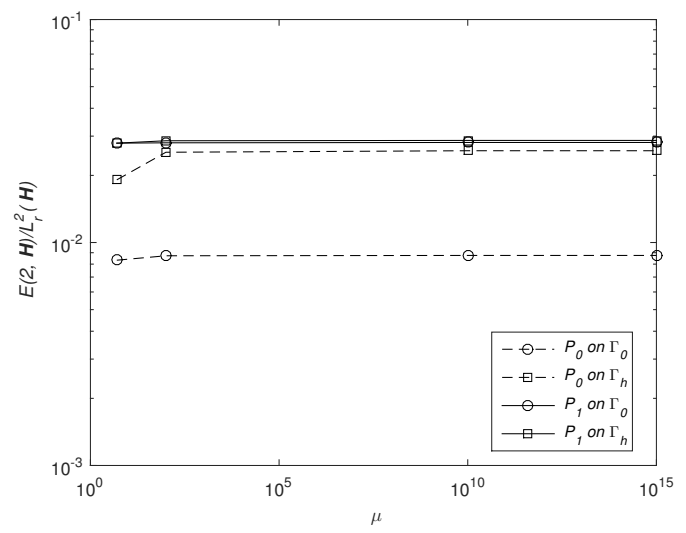

Figure 2. The relative error of the approximate boundary field versus the relative permeability.

the region of stable accuracy. The loss in accuracy for increasing $\mu$ have been observed in [2], where the stability was improved up to $\mu=10^{19}$ by a modified evaluation of the magnetic field.

\section{Conclusions}

Let us finally conclude the main results and observations.

The exterior transmission problem $(2.1),(2.2),(2.4)$ for the electro- or magnetostatic potential allows the reformulation as two integral equations in the meridian plane for the boundary potential and the boundary flux, which can be solved successively. The second kind integral equation is formulated only for the potential. The first kind integral equation for the flux contains the potential only at the right-hand side. The axisymmetric collocation method for this problem statement shows numerical convergence and good accuracy.

We suggest to redefine the kernel functions for the source point at the symmetry axis. It results in a non-singular asymptotic behavior of kernels for integration points, tending to the symmetry axis. The redefined kernels of the axisymmetric collocation method on smooth and polygonal boundaries are weakly singular.

According to the test computations, the polygonal boundary interpolation does not change the convergence behavior on the smooth boundary for the piecewise-constant and the piecewise-linear collocation on uniform meshes. The convergence order of the axisymmetric collocation coincides with the theoretically predicted results for $2 \mathrm{D}$-collocation. The $L_{r}^{2}$-error for the $P_{0}$-collocation, computed only at the collocation points by the midpoint rule, shows a one order higher convergence than the theory predicts. The $P_{1}$-collocation on the polygonal boundary shows a decrease of the convergence order by one if the solid angle is not included into the integral formulation. 


\section{Acknowledgments}

The authors want to thank the Belarusian Republican Foundation for Fundamental Research for financial support of the project 1.5.03.2 within the State Research Program "Convergence". We thank the referees for providing the constructive comments and helpful suggestions, which significantly contributed to improving the quality of the publication. In particular, we are very grateful for the comment on the correct treatment of the jump term in boundary integral equations on piecewise-smooth boundaries.

\section{References}

[1] M. Abramowitz and I.A. Stegun. Handbook of mathematical functions. Dover, New York, 1965.

[2] Z. Andjelic, G. Of, O. Steinbach and P. Urthaler. Fast boundary element methods for industrial applications in magnetostatics. In U. Langer, M. Schanz, O. Steinbach and W.L. Wendland(Eds.), Fast Boundary Element Methods in Engineering and Industrial Applications, volume 63 of Lecture Notes in Applied and Computational Mechanics, pp. 111-143, Berlin, 2012. Springer Berlin Heidelberg. http://dx.doi.org/10.1007/978-3-642-25670-7_4.

[3] D.N. Arnold and W.L. Wendland. The convergence of spline collocation for strongly elliptic equations on curves. Numerische Mathematik, 47(3):317-341, 1985. http://dx.doi.org/10.1007/BF01389582.

[4] L. Bernardin, P. Chin, P. DeMarco, K.O. Geddes, D.E.G. Hare, K.M. Heal, G. Labahn, J.P. May, J. McCarron, M.B. Monagan, D. Ohashi and S.M. Vorkoetter. Maple 15. Maplesoft, a division of Waterloo Maple Inc., Waterloo, Ontario, 2011. ISBN 978-1-926902-08-1.

[5] R.A. Białecki, U. Herding, O. Köhler and G. Kuhn. Weakly singular 2D quadratures for some fundamental solutions. Engineering Analysis with Boundary Elements, 18(4):333-336, 1996. http://dx.doi.org/10.1016/S0955-7997(97)000015.

[6] R. Buchau, W. Hafla and W.M. Rucker. Accuracy investigations of boundary element methods for the solution of Laplace equations. Magnetics, IEEE Transactions on, 43(4):1225-1228, 2007. http://dx.doi.org/10.1109/TMAG.2007.892304.

[7] M. Costabel and E.P. Stephan. On the convergence of collocation methods for boundary integral equations on polygons. Math. Comp., 49(180):461-478, 1987. http://dx.doi.org/10.1090/S0025-5718-1987-0906182-9.

[8] J. Elschner and I.G. Graham. An optimal order collocation method for first kind boundary integral equations on polygons. Numerische Mathematik, 70(1):1-31, 1995. http://dx.doi.org/10.1007/s002110050107.

[9] W. Hackbusch. Integral equations: theory and numerical treatment. Birkhäuser Basel, 1995. http://dx.doi.org/10.1007/978-3-0348-9215-5.

[10] L.D. Landau and E.M. Lifshitz. Electrodynamics of Continuous Media (translated from the Russian by J. B. Sykes and J. S. Bell). Pergamon Press, Oxford, 1960.

[11] O. Lavrova. Numerical methods for axisymmetric equilibrium magnetic-fluid shapes. PhD thesis, Fakultät für Mathematik, Otto-von-Guericke Universität Magdeburg, Germany, 2006. 
[12] O. Lavrova, G. Matthies, T. Mitkova, V. Polevikov and L. Tobiska. Numerical treatment of free surface problems in ferrohydrodynamics. Journal of Physics: Condensed Matter, 18(38):2657-2669, 2006. http://dx.doi.org/10.1088/09538984/18/38/S09.

[13] O. Lavrova, V. Polevikov and L. Tobiska. Instability of a magnetic fluid drop in a capillary: A numerical study. Magnetohydrodynamics, 44(2):3-9, 2008.

[14] P. Minciunescu. Contributions to integral equation method for 3D magnetostatic problems. Magnetics, IEEE Transactions on, 34(5):2461-2464, 1998. http://dx.doi.org/10.1109/20.717566.

[15] Nam-Trung Nguyen, A. Beyzavi, K.M. Ng and X.Huang. Kinematics and deformation of ferrofluid droplets under magnetic actuation. Microfluid Nanofluid, 3(5):571-579, 2007. http://dx.doi.org/10.1007/s10404-007-0150-y.

[16] R. Rannacher and W.L. Wendland. On the order of pointwise convergence of some boundary element methods. part ii: Operators of positive order. Mathematical Modelling and Numerical Analysis, 22(2):343-362, 1988.

[17] R.E. Rosensweig. Ferrohydrodynamics. Dover Pubns, 1997.

[18] D. Shi, Q. Bi, Y. He and R.Zhou. Experimental investigation on falling ferrofluid droplets in vertical magnetic fields. Experimental Thermal and Fluid Science, 54:313-320, 2014. http://dx.doi.org/10.1016/j.expthermflusci.2014.01.010.

[19] A. Sutradhar, G.H. Paulino and L.J. Gray. Symmetric Galerkin Boundary Element Method. Springer Berlin Heidelberg, 2008. http://dx.doi.org/10.1007/9783-540-68772-6. 